\title{
Reliable data collection algorithm based on AUVS online prediction
}

\begin{abstract}
The communication process in Underwater Acoustic Sensor Networks (UASNs) is susceptible to interference, which leads to higher latency and Bit Error Ratio (BER). The Autonomous Underwater Vehicle (AUV) polling to collect data has been used to provide reliable transmission of data and extend the network lifecycle. The target events underwater are random appeared. In order to effectively capture the target event, an algorithm for the AUV polling to collect data with online prediction is proposed, namely RCAP (Reliable Collection based on AUV with Prediction). Firstly, the polling objects to AUV should be determined. The clustered network structure is established. The cluster head will be polled by the AUV to transfer the data from the cluster. Secondly, the forecast model is build. The first $\mathrm{N}$ rounds of data collected are the historical data. Using the historical data, the data of the $\mathrm{N}+1$ th round is predicted based on the regression method. Then, the $\mathrm{N}+1$ to $2 \mathrm{~N}$ rounds of data are used to calibrate the predicted values, and the prediction model is optimized. Finally, the AUV polling trajectory would be plan. All the cluster head have storage threshold. According to the data volume prediction of each cluster head, the AUV polling objects of each round can be determined to maximize the amount of data collection and to improve network efficiency. The simulation results show that the algorithm can adapt to the prediction of target events in a variety of distributions. Especially, when the target events obey the linear distribution, compared with AAEERP, RCAP has a great optimization in network energy consumption, throughput, data transmission efficiency. It increases nearly $10 \%$ throughput especially.
\end{abstract}

Keywords: Underwater acoustic sensor networks(UASNs); Polling for data collection; online prediction; Clustering interaction
Volume 2 Issue 5 - 2017

\author{
Chen Qiuli, ${ }^{1,2}$ He Ming, ${ }^{1,2,3}$ Dai Fei,' Shen \\ Jian, ${ }^{2}$ Zhou Bo' \\ 'College of Command Information System, PLA Science and \\ Technology University, China \\ ${ }^{2}$ Nanjing University of Information Science and Technology, \\ China \\ ${ }^{3}$ The 6 I th Research Institute of PLA, China
}

Correspondence: He Ming, College of Command Information System, PLA Science and Technology University, China, Email paper_review@I26.com

Received: March 27, 2017 | Published: June 22, 2017
Abbreviations: UASNs, underwater acoustic sensor networks; BER, bit error ratio; AUV, autonomous underwater vehicle; RCAP, reliable collection based on auv with prediction.

\section{Introduction}

The underwater acoustic sensor networks (UASNs) is a kind of new marine measurement and control technology that combines autonomous data acquisition, data fusion and transmission applications in oceanographic data collection, water pollution monitoring, earthquake and tsunami prediction, marine navigation and underwater military surveillance, enemy target tracking and other aspects of the potential application value. So that the military and scientific research departments attach great importance to it. In recent years, China has proposed the strategic requirements of building a strong marine power, and the key technologies of the underwater acoustic sensor network are listed as the key research directions. The related researches mainly focus on the fields of MAC protocol, routing protocol, clock synchronization location, target recognition tracking and so on. However, the main problem affecting the performance of the underwater acoustic network is that the energy of the underwater nodes is limited and easy to disappear. Although the current research scholars have adopted a variety of routing protocols to minimize node energy consumption, such as the choice of the next hop node, the node between the rotation of the cluster head to balance the energy, the communication between the network nodes is still multi-hop transmission, and relay nodes are always more likely to consume energy and to be failure, based on multi-hop routing energy-saving breakthrough. Some scholars have proposed to use AUV to load energy and make supplement to the sensor nodes on a regular basis, although it is feasible, the node energy acquisition process is difficult to control by man-made control and cannot guarantee the sensor node long-term normal work. In addition, the harsh and unpredictable underwater application environment poses a significant challenge to routing tasks. ${ }^{1-3}$

In order to effectively and reliably collect data, jump out of the whole network node multi-hop transmission architecture, research scholars have proposed a mobile gateway to collect data. ${ }^{4,5}$ In this method, the AUV is used as the mobile gateway, and the corresponding sensor nodes are polled periodically to collect the relevant perceptual data. This method can effectively reduce the energy consumption of the sensor nodes, and AUV interact with the sensor nodes when the distance is close with reliable communication, effectively reducing packet loss rate and improving the network life cycle. In these schemes, there are multiple types of polling paths designed to improve the efficiency of data collection. Some researchers discuss the different polling objects, such as polling the cluster heads, random polling and so on. While some researchers discuss the different AUV polls structures, which are divided into level polling and vertical polling. The objective of our paper is to propose a predictive online learning polling model. With the random occurrence of underwater target events, the AUV can continue to learn and predict, and reasonably plan the polling process in the polling process, so as to increase polling in the target event aggregation area, reduce polling in the target event sparse, and perceive data less region. So that the data collection efficiency can be improved. The model can be applied to a variety of existing polling architectures, with good adaptability and promotion. The organization structure of the remaining part of this paper is as follows: the Section 2 of this paper discusses the related work and the existing problems; the Section 3 of this paper describes the online learning prediction model; in Section 4 of this 
paper, the validity and rationality of the model proposed in this paper is validated through contrast experiments, and conduct performance evaluation. The full text is summarized and prospected in Section.

\section{Related work}

For the underwater complex tasks, it is necessary to have fixed nodes to monitor the target area in real time, and the mobile nodes are required to dynamically capture the abnormal state. Therefore, the three-dimensional heterogeneous dynamic model becomes the mainstream model of the current underwater network operation and maintenance. Taking into account the cost of AUVs, there is only a small number of AUVs are deployed in network, most of them are the ordinary sensor nodes. As the AUVs function is strong and energy is high, it has a good effect in the aspect of the reliable transmission of data. To this end, the researchers put forward a series of AUVs auxiliary underwater acoustic sensor networks data reliable collection algorithm, only the AUVs mobility is used to collect data information to the ordinary node polling. The network architecture can be roughly divided into horizontal polling and vertical polling and as shown in Figure 1.

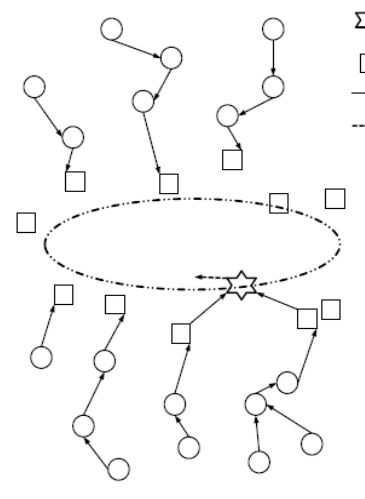

(a) horizontal polling architecture

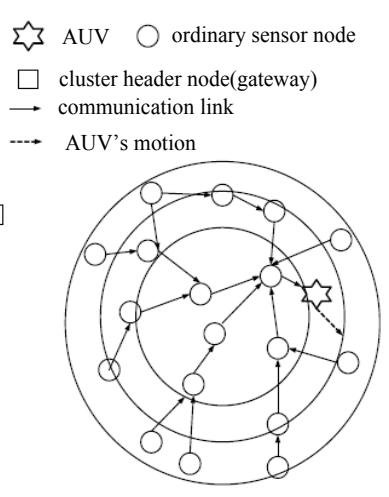

(b) vertical polling architecture
Figure I Reliable Collection Architecture based AUV in Underwater Acoustic Sensor Networks. 6.13

Initially, the horizontal polling architecture is aimed at a twodimensional network of sensor nodes at the bottom. AEERP ${ }^{6}$ uses a single AUV to interact with the underlying gateway. The bottom gateway node is replaced by a randomly selected method and set the energy consumption threshold. The shortest path tree construction method is used in other nodes to connect with the nearest gateway to generate the network topology. This method effectively reduces the number of hops of the data transmitted by the underwater nodes, reduces the error codes caused by the attenuation, and ensures the integrity and reliability of the data. Based on the horizontal polling, $\mathrm{AURP}^{7}$ constructs a number of AUVs polling architectures for the first time and designs the elliptical trajectory, and uses heterogeneous acoustic communication channel. Three kinds of data transmission methods are designed according to the distance, which can reduce the same frequency interference with each other. $\mathrm{Khan}^{8}$ proposed a hierarchical clustering structure and the bottom nodes are divided into three categories of underwater gateway node, path node and ordinary node. The underwater gateway node is the cluster head, and the path nodes on the AUV polling path that will be interactive, and the ordinary node is used as an alternative to replace the energy node with too large energy consumption. The AUV greatly increases the transmission rate of the packet and reduces the overall energy consumption by interacting with multiple path nodes. However, the algorithm needs to divide the monitoring sea area according to the number of underwater gateway nodes. The preprocessing process is cumbersome, and the selection of multiple path nodes is controlled by the underwater gateway nodes, and the higher performance of the gateway node is required.

TCM algorithm ${ }^{9}$ is suitable for dynamic 2D underwater environment. The particle swarm optimization algorithm is used for the bottom nodes to cluster. The horizontal polling way is used by AUV to interactively access dynamic cluster head. Although it is more suitable for dynamic underwater environment, TCM algorithm still has many disadvantages. For example, the cluster head changes frequently, it need to constantly notify the AUV node new cluster head ID. Then, the network energy consumption increased. In Shah, ${ }^{10}$, the horizontal architecture can only be deployed by hierarchical in 3D environment. Each layer has a separate AUV polling to achieve reliable data collection and forwarding. To this end, a vertical polling architecture of the polling method is proposed in Umar. ${ }^{11}$ By the definition of the depth of the node, the AUV vertical motion is used to transfer the data from the high depth region to the low depth region. For the three-dimensional dynamic underwater environment, the LVRP algorithm Shi ${ }^{12}$ is used and the gateway selection according to the Voronoi formed among the nodes. It can effectively improve network performance by combining with AUV vertical polling.

The RE-AEDG algorithm Liaqat ${ }^{13}$ compares the horizontal and vertical polling and combines them together. In RE-AEDG, the underwater nodes randomly deployed are divided into five layers, and the nodes at the second layer and the fourth layer are the gateway nodes, and the nodes at the same layer do not communicate with each other. The nodes at the first, third and fifth layers are used to delivery data by selecting the nearest gateway according to the distance. The AUV vertical oval polling in the second and fourth layers is to achieve reliable data collection. However, the method requires that the surface nodes send the data to the water gateway, and then returned to the surface by the AUV, which will result in energy waste. As the two-dimensional network for the level of polling method is more mature, there are more ways to optimize this. AAEERP Ilyas ${ }^{14}$ has been improved on the basis of AEERP, and it is considered that the staying time of AUVs in each gateway should be different. Because the shortest path trees generated by different underwater gateway nodes are not the same, the more member nodes are, the more data should be collected. Therefore, the staying time of the AUV should be proportional to the number of nodes of the gateway members. Compared to the AEERP algorithm, AAEERP has lower power consumption and higher data collection capabilities.

AEDG Javaid ${ }^{15}$ discusses the elliptical trajectory of AUV horizontal polling. According to the selection area of underwater gateway nodes, the radius parameter of ellipse is discussed, which can be used to optimize the AUV polling trajectory based on changes in the gateway. Kartha ${ }^{16}$ discusses the polling trajectories of AUV in different scenarios from the perspective of delayed tolerances, including square polling, helical polling, and elliptical polling and so on. A network data collection framework is effectively established for different situations, and can be used for more flexible implementation of different service strategies. In Khan, ${ }^{17}$ four AUVs interactive polling optimization algorithms are designed on the basis of hierarchical 
clustering structure in Khan. ${ }^{8}$ The method is defined to share the state information of four AUVs. This method not only realizes the data collection and transmission to the water gateway, but also can help communicate data between the two-dimensional layer nodes through the cooperative communication between the four AUVs. In Dalal, ${ }^{18}$ a scalable data encryption and decryption algorithm is designed based on AURP for underwater safety challenges. Different AUVs are used for different monitoring waters, and each AUV collects data relying with the gateway node matching key of its monitoring waters. This method guarantees the network security and optimizes the network performance.

To sum up, the existing polling method mainly has the following problems:

a. Most of the existing polling architectures are deployed according to two-dimensional plane of sensor nodes. There are few methods for three-dimensional space polling with large loopholes and are not suitable for large-scale promotion.

b. The polling trajectory of the AUV is fixed. Even if there are literature Kartha ${ }^{16}$ focused on the merits of different polling trajectories, the various trajectories used in the network life cycle are fixed. The fixed trajectory can't adapt to the dynamic evolution of underwater network. It is difficult to ensure the reliability of data communication after the replacement of the interactive nodes, and cannot guarantee the efficiency of data collection at all times.

c. The randomness of the target event is not considered. The data collection across the entire network should be considered in the existing polling architecture, and the networks deployed in most of water areas are targeted. In order to extend the life of the network and reduce the energy consumption of the nodes, it is necessary to collect the monitoring data of the target events and collect the information of the whole network, which not only enhances the energy consumption of the node, but also makes the processing of the subsequent data more complicated.

d. The AUV energy consumption problem is not considered. It is assumed that AUV nodes are infinite energy in most of articles, regardless of their energy consumption problems in the network. Most of the algorithms are designed to sacrifice AUV energy consumption in exchange for the life of ordinary sensor nodes. Although AUV energy is several orders of magnitude compared to ordinary sensor nodes, there are still energy limits, and the energy which is assumed infinite is not realistic.

In view of the above problems, this paper analyzes the shortcomings of the current polling architectures, and proposes the AUV online learning polling trajectory prediction model, which can be applied to any of the above structures to improve the network data collection performance.

\section{RCAP model based on time series analysis}

\section{Preparation work}

Firstly, we introduce the function definition of each node in the network:

Interactive nodes: the nodes in the network are clustered and the cluster head nodes are used as the interaction nodes. They are used for collecting the perceived data of other nodes in the cluster and delivering them to the AUV nodes.
Ordinary nodes: they are used for monitoring the target events in the network and delivering the perceived data to the cluster head interaction nodes of the cluster itself.

AUV: it is used for polling the interactive nodes in the network, and collects and delivers it to the gateway nodes on a regular basis.

This section will introduce the online learning trajectory prediction model. In order to facilitate the analysis and solution of the model, the following assumptions are given under the premise of reliability in line with the actual application scenarios:

Assumption 1: each node knows its location information after deployment, and the AUV has the autonomous positioning function.

Assumption 2: AUV energy is much larger than the sensor node not unlimited, regardless of its failure in the process of operation.

Assumption 3: the sensor node has a certain capacity of store.

(Figure 2) The key to collect data by AUV polling lies in two points: one is what nodes to be polled; the other is how to poll these nodes. In the case of question 1, the existing literature generally deals with the established trajectory of the AUV, and the nodes near the trajectory are set as the polled node. The remaining nodes in the small-scale network can transmit the self-perceived data to the other Inquiry node by mean of near transmission. Large-scale networks are clustered depending on the nodes being polled, and the remaining nodes transmit the perceived data to the cluster heads to achieve AUV node on the whole network data collection. In the case of question 2, the repetition traversal method is used in most of existing literatures, which repeated polling along the established trajectory, until the end of the acquisition task and no data aggregation. Since the time interval exists in AUV node polling, the data collected by AUV from the same node at a time can form a set of sequences. Due to the uncertainty of the underwater environment, the target events monitored by each region are not the same, and the number of packets perceived and collected is different. There are many packets of AUV to be delivered by some interactive nodes, and the few packets are delivered by other interactive nodes. Therefore, the uniform traversal of each node to be interactive not only can reduce the efficiency of AUV polling, but also increase the packet delay. Therefore, this paper designs the online learning prediction model based on time Series Analysis, learns the historical data of the pre-traversal of the AUV, and makes the prediction of the number of interactive nodes in subsequent polling and determines to carry out the interaction according to the size of the predicted data node polling.

In this paper, the prediction polling is divided into two parts. The first part is the generation of the prediction model and the other part is the planning of the AUV polling trajectory. The number and location of the interaction nodes are determined using the method specified in literature. ${ }^{11}$ After the cluster, there is a total of $\mathrm{N}$ and expressed as $S$, and the set of all the nodes is $S=\left\{s_{1}, s_{2}, s_{3} \ldots s_{n}\right\}$. The time interval of the data collection is set to be $T$, that is, AUV polling is done every $\mathrm{T}$ time, and the data of the interactive node shall be collected. The interactive node is used for tagging the first packet as $T_{j}$ within each interval. $x_{i j}$ means the amount of data collected by the $s_{i}$ node during the $\mathrm{j}$ time. The number of packets generated by the $s_{1}$ node in the $T_{1}$ time period is $x_{11}$.

\section{Generation of prediction model}

Since the model is used to predict historical data required to be used, it is assumed that AUV is used in the whole network polling 
in the first ten times. The sliding window is used for the selection of the historical data, and the size of the sliding window is initially set to be 5 . That is, using the historical data of 1 to 5 of time periods to generate the prediction model, and slide five times and use 6 to 10 of time periods to modify the model to determine the prediction model Figure 3.

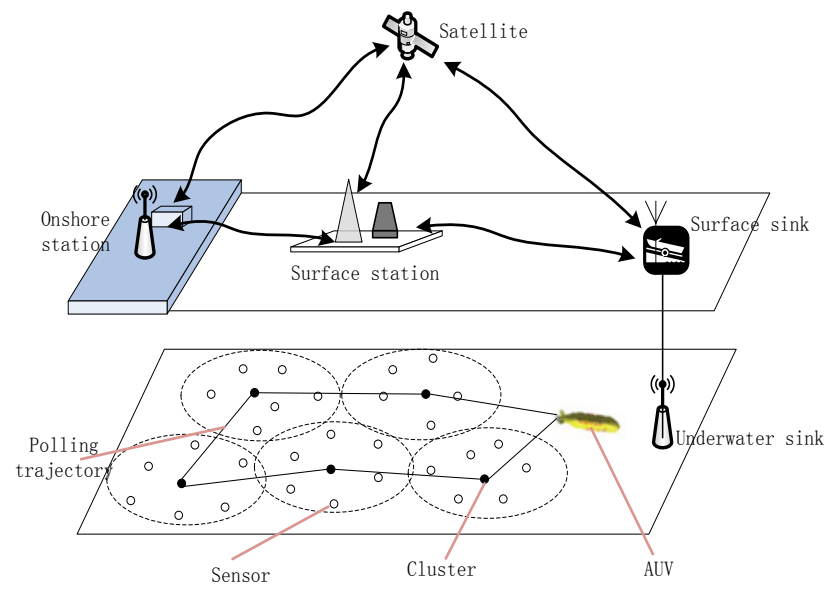

Figure 2 Polling model schematic.

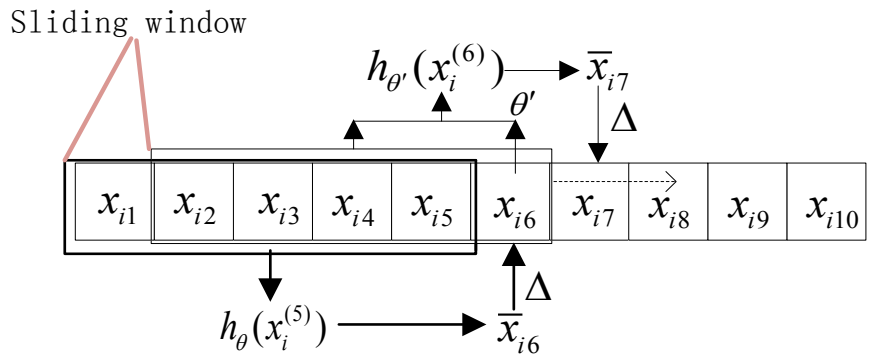

Figure 3 Sliding window schematic.

For node $N_{i}$, the amount of data packet collected in the first five time intervals is $x_{i 1}, x_{i 2}, x_{i 3}, x_{i 4}, x_{i 5}$. The predictive vector is $\theta, \theta=\left(\theta_{1}, \theta_{2}, \theta_{3}, \theta_{4}, \theta_{5}\right)^{T}$. And it is used to adjust the influence of each component the historical data on the late data prediction. The $N_{i}$ amount of data in the sixth time period can be predicted by the following formula:

$$
\bar{x}_{i 6}=\left(\theta_{1} x_{i 1}+\theta_{2} x_{i 2}+\theta_{3} x_{i 3}+\theta_{4} x_{i 4}+\theta_{5} x_{i 5}\right)=\theta^{T} X^{(5)}
$$

General expression of the estimation function in the prediction model is as follows:

$$
h_{\theta}\left(x_{j}\right)=\theta^{T} X^{(j-1)}
$$

Since the length of the sliding window is set to be 5 , the $X^{j-1}$ is used to express the vector consisting of five window data up to $x_{j-1}$, i.e $X^{j-1}=\left(x_{j-5}, x_{j-4}, x_{j-3}, x_{j-2}, x_{j-1}\right)$

Due to the random generation of initial prediction vector $\theta$, the gradient descent model is used to calibrate the predictive variables in order to make the late prediction more accurate. The actual packet of $s_{i}$ node collected at the $T_{6}$ time period is $x_{i 6}$, and the prediction error is:

$$
\begin{aligned}
\Delta & =\mathrm{x}_{i 6}-\overline{\mathrm{x}}_{i 6} \\
& =\mathrm{x}_{i 6}-\mathrm{h}_{\theta}\left(\mathrm{x}_{6}\right) \\
& =\mathrm{x}_{i 6}-\theta^{\mathrm{T}} \mathrm{x}^{(5)}
\end{aligned}
$$

The error function of $J_{j}(\theta)$ can be used to describe the pros and cons of the estimation function of $h_{\theta}\left(x_{j}\right)$. The expression of the error function is:

$$
J_{j}(\theta)=\frac{1}{2}\left(h_{\theta}\left(x_{j}\right)-x_{j}\right)^{2}=\frac{1}{2}\left(\theta^{T} X^{j-1}-x_{j}\right)^{2}
$$

In order to minimize the value of the error function to get $\min J_{\theta}$ , the location where the gradient of the function decreases most fast, that is, the partial derivative of the function can be expressed as:

$$
\begin{aligned}
& \frac{\partial}{\partial \theta} J(\theta)=\frac{\partial}{\partial \theta} \frac{1}{2}\left(h_{\theta}\left(x_{j}\right)-x_{j}\right)^{2} \\
& =\frac{\partial}{\partial \theta} \frac{1}{2}\left(\theta^{T} X^{j-1}-x_{j}\right)^{2} \\
& =\left(\theta^{T} X^{j-1}-x_{j}\right)^{2} X^{(j-1)}
\end{aligned}
$$

And then the prediction vector of $\theta$ is updated and the vector $\theta$ is reduced in the direction of the smallest gradient. The updated $\theta^{\prime}$ can be expressed as:

$$
\begin{aligned}
\theta^{\prime} & =\theta-\alpha \frac{\partial}{\partial \theta} J(\theta) \\
& =\theta-\alpha\left(\theta^{T} X^{(j-1)}-x_{j}\right) X^{(j-1)}
\end{aligned}
$$

Where $\alpha$ refers to step size, i.e. the variable quantity is changed with the direction of gradient reduction each time. As the gradient is directional, for vector of $\theta$, a gradient direction can be solved for each component, so that a whole direction can be solved. At the time of the change, the function is changed towards the direction of the most down to reach a minimum point, which is to ensure the minimum error. It can be described in a simpler mathematical language, namely:

$$
\nabla_{\theta} J=\left[\begin{array}{l}
\frac{\partial}{\partial \theta_{j-5}} J \\
\cdots \\
\frac{\partial}{\partial \theta_{j-1}} J
\end{array}\right]
$$

$$
\theta=\theta-\alpha \nabla_{\theta} J
$$

Wherein $\nabla$ refers to gradient. When $J$ is equal to 6 to 10 , five verifications can be made separately to the $h_{\theta}\left(x_{j}\right)$ to obtain a more effective predictive value, which can be used to predict the data packet that may be generated by the late interactive nodes, so as to plan the reasonable polling trajectory of $\mathrm{AUV}$, and realize the maximization of data collection efficiency and minimize the delay.

\section{Planning of AUV polling trajectory}

Through the prediction model in the section 3.2 , it is possible to effectively estimate the number of packets aggregated by each interactive node in the next period of $T$. According to assumption 3, the sensor node has a certain storage capacity and the value is set to be $s_{i}$, when the amount of data generated by $s_{i}$ node in the current 
period of $T$ time is few, the point is polled by AUV and the harvest is low, which increases the network delay.

The steps of AUV polling trajectory planning strategy proposed in this section are as follows:

Step 1: the prediction model is used to estimate the amount of data packets generated by two consecutive periods of $T$ for each interactive node. Determine if the node storage threshold of $C_{N}$ is exceeded.

Step 2: if it is predicted that the sum of data packets volumes generated by interaction node of $s_{i}$ during the two time intervals of T is over the storage threshold, it indicates that the AUV must poll the node; otherwise it will cause packet loss. The node of $s_{i}$ is included in the path planning considerations.

Step 3: if it is predicted that the sum of data packets volumes generated by interaction node of $s_{i}$ during the two time intervals of $\mathrm{T}$ is not over the storage threshold, it is not necessary to traverse the node. The node will not be considered in the path planning.

Step 4: make statistics of the polling nodes, and develop a reasonable planning route according to the location of each node.

Step 5: after traversing the selected node, the estimated function is continually calibrated using the new round of data collected.

Step 6: if the node of $s_{i}$ has not been included in the path plan after the prediction for twice, it will be direct traversal at the third time without predicting (Figure 4).

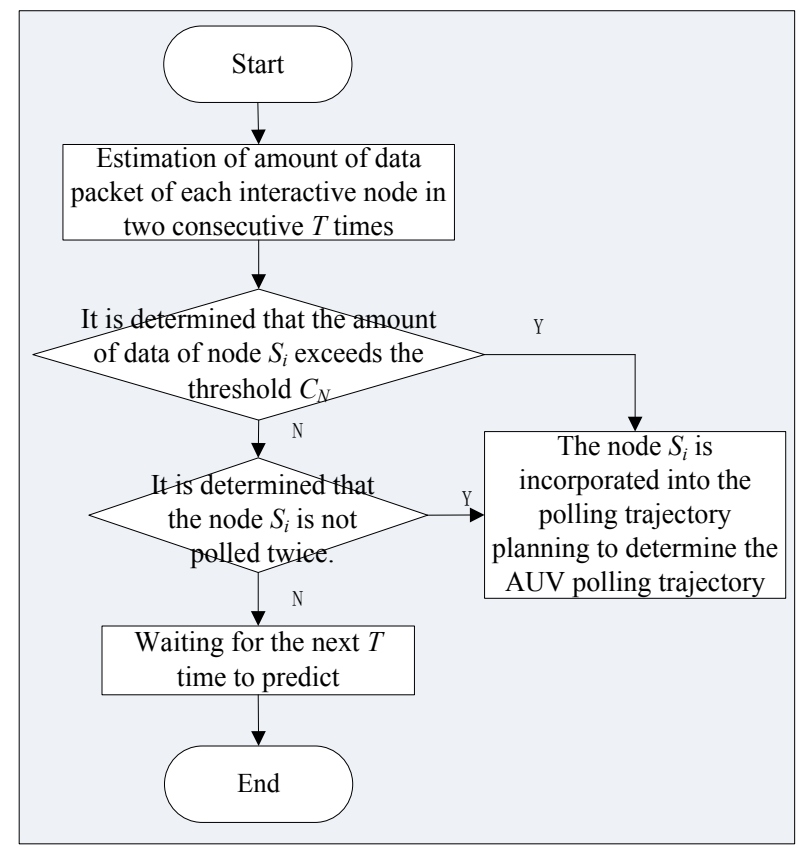

Figure $4 \mathrm{AUV}$ polling trajectory planning flow chart.

\section{Simulation experiment}

In order to validate the versatility and validity of the model, the model is applied to the representative AAEERP ${ }^{14}$ horizontal polling architecture, and 4 groups of experiments are designed with its original model for comparison from sensor node energy consumption, network throughput, packet transmission rate, end-to-end delay design: a) Energy consumption: during the network monitoring, the total amount of energy consumed by all sensor nodes is mainly for data transmission and reception. The energy consumption unit is Joule (J).

b) Throughput: refers to the amount of data transferred from sender to receiver. The network throughput has directly affects on the number of nodes in the network for data transmission and the duration of that number. The unit of throughput is bit.

c) Packet delivery ratio: refers to the ratio of data packet successfully transmitted to the water gateway by AUV to the total amount of data packet generated by the network.

d) End-to-end delay: refers to the total time that the data packet is transmitted to the water gateway. The unit is sec (s).

According to the experimental parameters of the horizontal polling architecture AAEERP, ${ }^{14}$ the compared parameters of the simulation experiment are as follows: the underwater sensor nodes and AUV are deployed in the $1500 \mathrm{~m} * 2000 \mathrm{~m}$ area. A different number of sensor nodes are deployed to demonstrate the utility of the data collection algorithm in the underwater acoustic sensor networks of different sizes. It is assumed that UWSNs have different numbers of sensor nodes of 18, 30, 42, 54 and 64 ; the sensor node transmission range is $250 \mathrm{~m}$, and the initial energy is 70 joules and the size of each packet is 70 bytes. It is assumed that no collision exists between the underwater communication channels and the interference effects between the channels are ignored. The specific simulation parameters are as shown in Table 1. Considering the randomness of the underwater target monitoring event, the feasibility of the RCAP is verified firstly.

Table I Horizontal polling experimental parameters

\begin{tabular}{ll}
\hline Parameters & Values \\
\hline Monitoring range & $2000 \mathrm{~m}^{*} 1500 \mathrm{~m}$ \\
Number of nodes & $18,30,42,54,64$ \\
Initial energy of node & $70 \mathrm{~J}$ \\
Data collection factor & 0.6 \\
Size of data packet & 70 bytes \\
Node transmission range & $250 \mathrm{~m}$ \\
Number of AUVs & $\mathrm{I}$ \\
\hline
\end{tabular}

\section{Experiment I: Feasibility verification of reliable collection algorithm based on AUV with prediction}

The error rate of the data collection algorithm is based on the distribution of various target events, such as linear distribution, normal distribution and Poisson distribution. The experimental results are shown in Figure 5. The error rate of the data transmission is predicted in the case of various target events with the increase of the size of the network. For the target event of the linear distribution (the distribution of the event is subject to the AR model), the error rate of the prediction algorithm is kept at a low level, and the larger the network size is, the lower the predicted error rate is. This is because the time series analysis used in this paper is based on historical data to conduct iterative prediction, and it is a better trend forecast for the linear distribution. When the event in the network subjects to the normal distribution, the error rate of the algorithm is higher than that of the forecasting algorithm, but it tends to be stable with the increase of the network scale. Although it is not as accurate as the prediction 
of linear distribution events, as a whole, the prediction of events in large-scale networks can be less than $30 \%$, and the trend is convergent and has a certain of operability. For the Poisson distribution event, this paper predicts that the effect of the algorithm fluctuates greatly, the average error rate is about $50 \%$, and the trend diverges and the operability is not strong. To this end, the simulation experiment of data collection algorithm is carried out under the premise of linear distribution and normal distribution of target events. The experiments numbered from 2 to 5 are the comparison of energy consumption, throughput, transmission rate and time delay for the AAEERP horizontal polling architecture.

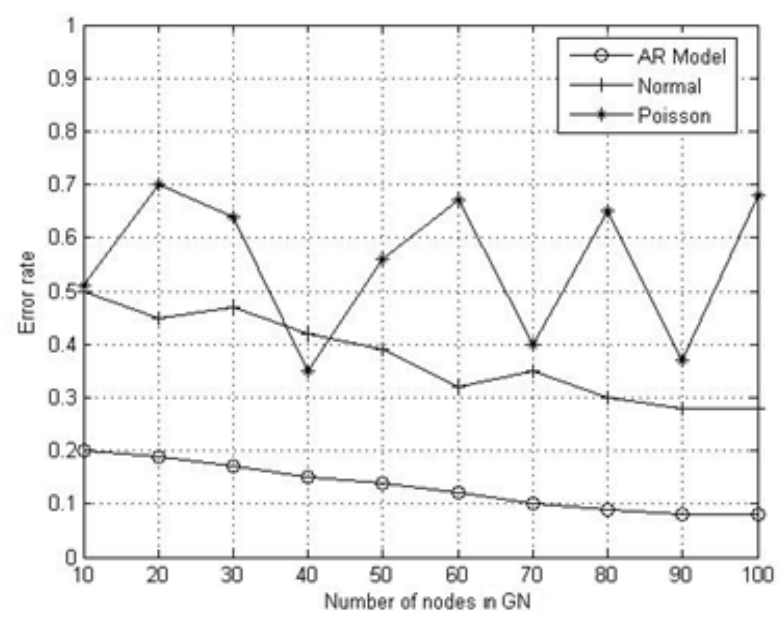

Figure 5 Error rate comparison of predictive algorithms in different event distribution.

\section{Experiment 2: Comparison of network energy consumption}

AAEERP algorithm and the algorithms specified in this paper are used for data transmission under the circumstances of different event distribution, and the total energy consumption of the entire network is calculated. The data results are shown in Figure 6. It can be seen from Figure 6 that the RCAP (AR) prediction algorithm is more accurate and the energy consumption is lower for the target event with linear distribution. Compared with the AAEERP algorithm which follows the elliptical motion, the AUV trajectory designed in this paper is more flexible and the number of unnecessary polling is reduced to effectively improve the efficiency of data collection. In case of the target event of normal distribution, since RCAP (normal) prediction effect is relatively poor, compared to linear prediction, the energy consumption is greater.

\section{Experiment 3:Throughput comparison}

AAEERP algorithm and the algorithms specified in this paper are used for data transmission under the circumstances of different event distribution, and the total amount of the data packet transmitted from source mode to destination node is calculated. The data results are shown in Figure 7. Figure 7 shows the throughput of each algorithm. It can be seen that under the target event of linear distribution, the RCAP (AR) prediction algorithm is accurate, the data transmission efficiency is high, the throughput is increased with the increase of the network scale, and the gain is higher. For the AAEERP algorithm, the network throughput is at the middle level and tends to be stable and the rising space is small. For the target event with normal distribution, the RCAP (normal) prediction algorithm has a small increase in throughput, but the network throughput rises and the rising trend is obvious subsequently.

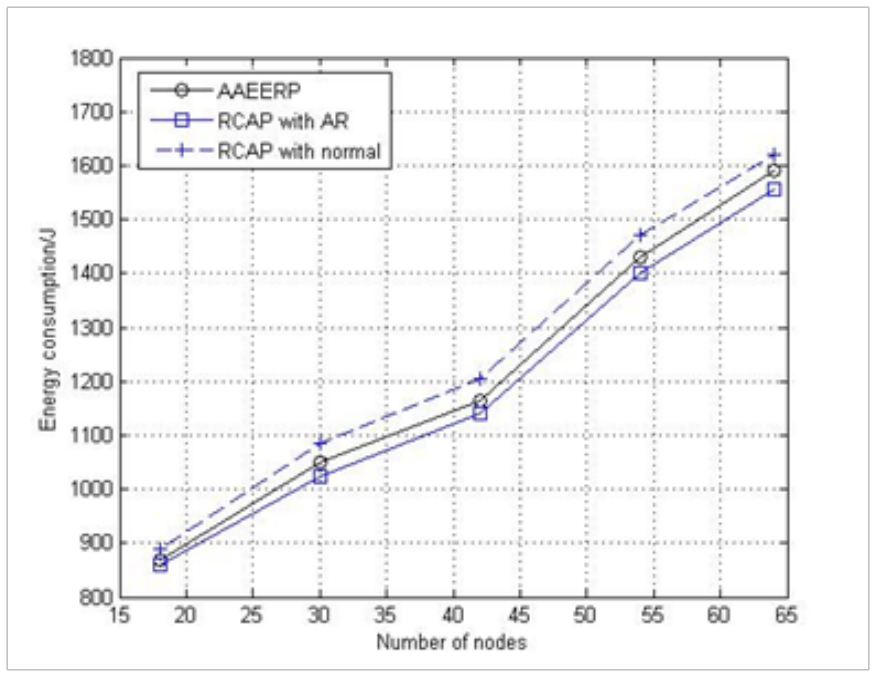

Figure 6 Comparison diagram of energy consumption.

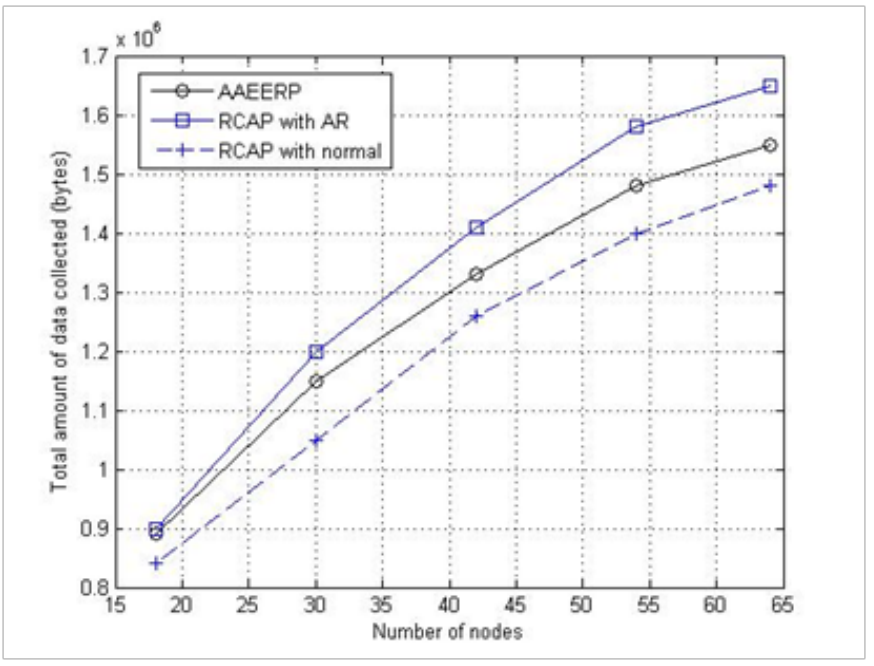

Figure 7 Comparison diagram of throughput.

\section{Experiment 4: Comparison of data packet delivery ratio}

AAEERP algorithm and the algorithms specified in this paper are used for data delivery under the circumstances of different event distribution, and the ratio of the data volume successfully delivered to the water gateway to the data volume actually generated in the network is calculated. The data statistics results are shown in Figure 8. It can be seen from Figure 8 that the data delivery ratio obtained by the RCAP (AR) prediction algorithm is always higher and the reduction ratio is slower for the target event with linear distribution. This is because when the node used for interacting with the AUV is polled, the algorithm can change the polling trajectory in time, while the AAEERP algorithm still moves in accordance with the elliptical trajectory, increasing the packet loss rate. The larger the scale of the network, the easier the change of the interaction node, and the difference in the transmission efficiency between the AAEERP algorithm and this algorithm will be larger. It further shows the usability of this algorithm in large-scale networks. 


\section{Experiment 5:Transmission delay comparison}

AAEERP algorithm and the algorithms specified in this paper are used for data delivery under the circumstances of different event distribution, and the total amount of the data packet transmitted from source mode to water surface gateway is calculated. The data results are shown in Figure 9. Figure 9 shows the end-to-end delay of the AEERP and RCAP algorithms. In the AUV auxiliary data collection algorithm, the end-to-end delay depends primarily on the round-trip time and speed of the AUV. The AUV polling trajectory is fixed by AEERP algorithm, the round-trip time and speed are relatively fixed, and the overall delay tends to be at a moderate level. And the RCAP (AR) prediction algorithm makes the track of AUV change with the amount of data in the network, and the number of AUV polling is increased with the number of packets generated by the interaction node. On the contrary, for some nodes with few data packets, although the efficiency of AUV data collection is greatly enhanced due to fewer interaction numbers, the data stored by it will have to wait for a long time to be collected and delivered by AUV for the data packet generation node, which increases the network end-to-end delay to a certain extent.

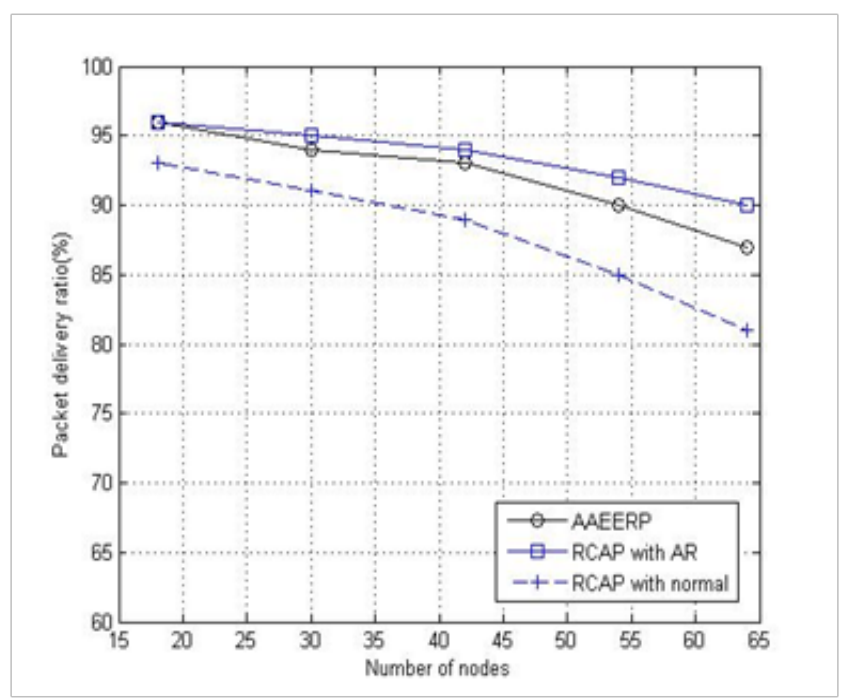

Figure 8 Comparison diagram of data packet delivery ratio.

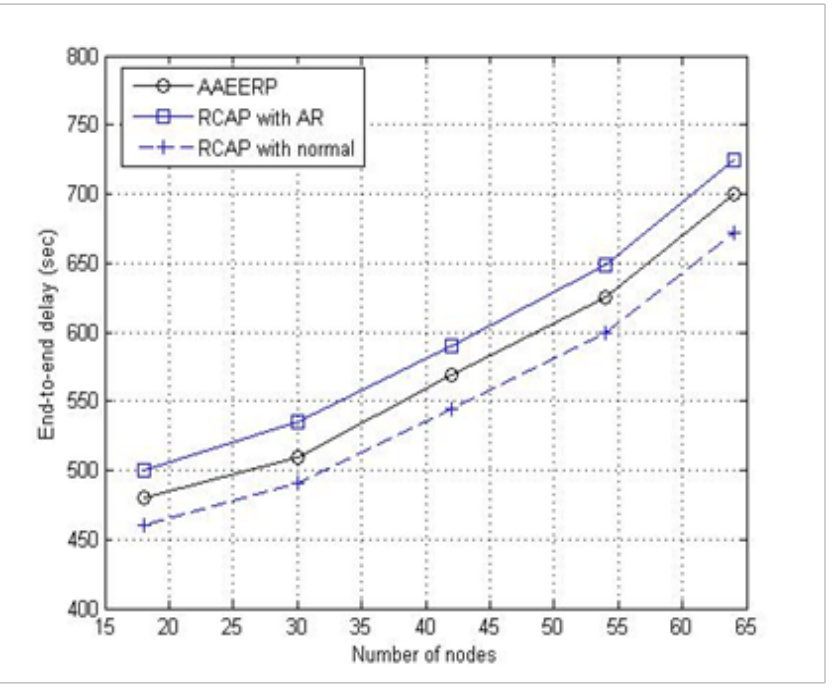

Figure 9 Comparison diagram of transmission delay.

\section{Conclusion and expectation}

In the current underwater acoustic sensor networks, the use of AUV polling to collect data has become an effective way to provide reliable transmission of data and extend the life cycle of the network. In this paper, a novel underwater polling method is proposed, namely RCAP (Reliable Collection based on AUV with Prediction). The AUV trajectory and its interaction time with each cluster head are scientifically developed, and the maximum number of propagation data and the minimization of the network delay are realized. The contributions of this paper are as follows:

a. The online prediction model for AUV polling is designed.

b. The effectiveness of RCAP is discussed under different distributions of underwater target events.

c. Compared with AEERP, it is verified that RCAP has certain advantages in network energy consumption, throughput and packet transmission rate, and has certain promotion value.

\section{Acknowledgments}

None.

\section{Conflict of interest}

Author declares that there is none of the conflicts.

\section{References}

1. Climent S, Sanchez A, Capella JV, et al. Underwater Acoustic Wireless Sensor Networks: Advances and Future Trends in Physical, MAC and Routing Layers. Sensors (Basel). 2014;14(1):795-833.

2. Yanhua Zhang, Xingming Sun, Wang Baowei. Efficient Algorithm for K-Barrier Coverage Based on Integer Linear Programming. China Communications. 2016;13(7):16-23.

3. He M, Liu F, Miao Z. A mechanism of topology optimization for underwater acoustic sensor networks based on autonomous underwater vehicles. International Journal of Distributed Sensor Networks. 2017;13(1):1-14.

4. Zhiguo Qu, John Keeney, Sebastian Robitzsch, et al. Multilevel Pattern Mining Architecture for Automatic Network Monitoring in Heterogeneous Wireless Communication Networks. China Communications. 2016;13(7):108-116.

5. Wang Y, Sun D, Fan W, et al. Low consumption dynamic time synchronization for mobile and high latency underwater acoustic communication networks. Ocean Acoustics (COA). 2016. p. 1-7.

6. Ahmad A, Wahid A, Kim D. AEERP: AUV aided energy efficient routing protocol for underwater acoustic sensor network. Proceedings of the $8^{\text {th }}$ ACM workshop on Performance monitoring and measurement of heterogeneous wireless and wired networks. 2013. p. 53-60.

7. Yoon S, Azad AK, Oh H, et al. AURP: An AUV-aided underwater routing protocol for underwater acoustic sensor networks. Sensors. 2012;12(2):1827-1845.

8. Khan JU, Cho HS. A Distributed Data-Gathering Protocol Using AUV in Underwater Sensor Networks. Sensors. 2015;15(8):19331-19350.

9. Zhang Y, Chen W, Liang J, et al. A Network Topology Control and Identity Authentication Protocol with Support for Movable Sensor Nodes. Sensors. 2015;15(12):29958-29969.

10. Shah P M, Ullah I, Khan T. MobiSink: Cooperative Routing Protocol for Underwater Sensor Networks with Sink Mobility. IEEE $30^{\text {th }}$ International 
Conference on Advanced Information Networking and Applications (AINA). 2016. p. 189-197.

11. Umar A, Javaid N, Ahmad A, et al. DEADS: Depth and Energy Aware Dominating Set Based Algorithm for Cooperative Routing along with Sink Mobility in Underwater WSNs. Sensors. 2015;15(6):14458-14486.

12. Shi L, Yao Z, Zhang B, et al. An efficient distributed routing protocol for wireless sensor networks with mobile sinks. International Journal of Communication Systems. 2015;28(11):1789-1804.

13. Liaqat T, Akbar M, Javaid N, et al. On Reliable and Efficient Data Gathering Based Routing in Underwater Wireless Sensor Networks. Sensors (Basel). 2016;16(9):1391.

14. Ilyas N, Javaid N, Iqbal Z, et al. AAEERP: Advanced AUV-Aided Energy Efficient Routing Protocol for Underwater WSNs. International Conference on Advanced Information Networking and Applications IEEE. 2015. p. 77-83.
15. Javaid N, Ilyas N, Ahmad A, et al. An Efficient Data-Gathering Routing Protocol for Underwater Wireless Sensor Networks. Sensors (Basel). 2015;15(11):29149-29181.

16. Kartha JJ, Jacob L. Delay and lifetime performance of underwater wireless sensor networks with mobile element based data collection. International Journal of Distributed Sensor Networks. 2015. p. 28.

17. Khan JU, Cho HS. Data-Gathering Scheme Using AUVs in LargeScale Underwater Sensor Networks: A Multihop Approach. Sensors. 2016;16(10):1626.

18. Dalal R, Singal MP, Agrawal V. Secure Co-operation of AURP (An AUV-Aided Underwater Routing Protocol) for Underwater Acoustic Sensor Networks. International Journal of Enhanced Research in Science Technology \& Engineering. 2015;4(5):123-127. 